\title{
Elevation of Mycobacterium tuberculosis subsp. caprae Aranaz et al. 1999 to species rank as Mycobacterium caprae comb. nov., sp. nov.
}

\section{Correspondence \\ Lucas Domínguez lucasdo@vet.ucm.es}

\author{
Alicia Aranaz, ${ }^{1}$ Debby Cousins, ${ }^{2}$ Ana Mateos ${ }^{1}$ and Lucas Domínguez ${ }^{1}$ \\ 'Departamento de Patología Animal I (Sanidad Animal), Facultad de Veterinaria, \\ Universidad Complutense, 28040 Madrid, Spain \\ ${ }^{2}$ Australian Reference Laboratory for Bovine Tuberculosis, Department of Agriculture, \\ South Perth 6151, Australia
}

Mycobacterium tuberculosis, Mycobacterium bovis, Mycobacterium africanum and Mycobacterium microti are the classical members of the M. tuberculosis complex. These pathogens, which affect both humans and animals, are closely related from a taxonomic point of view. Some phenotypic characteristics have been used for speciation within the M. tuberculosis complex. The fact that this group of organisms is important as pathogens of worldwide significance has led to bias in the selection of tests for the classification of the mycobacteria, with an enrichment of those tests most likely to separate them. This led to a distortion of the numerical taxonomic matrix (Wayne, 1984). Numerical taxonomy places the members of the complex in a macrocluster, as they have a close relationship to M. tuberculosis (sensu stricto) and they should be reduced to subspecies thereof (Wayne \& Kubica, 1986). DNA homology within the complex has raised some debate about the

Published online ahead of print on 1 May 2003 as DOI 10.1099/ ijs.0.02532-0.

Abbreviations: PZA, pyrazinamide; spoligotyping, spacer oligonucleotide typing. convenience of the reorganization of the complex as a single species. However, the subtle phenotypic differences, the epidemiology (preferred host) and pathology, and the ease of use of the classical names have kept the traditional nomenclature.

In relatively recent times, because of the increased research on tuberculosis, and coupled with the availability of new molecular tools, other isolates with characteristics of the $M$. tuberculosis complex, but not matching any of the classical species, have been described, i.e. 'M. canettii' (van Soolingen et al., 1997) and the seal bacillus (Cousins et al., 1990, 1993).

In 1999, we described the results of a polyphasic taxonomic study of an unusual member of the M. tuberculosis complex, and named this organism Mycobacterium tuberculosis subsp. caprae (Aranaz et al., 1999), because it was first isolated from goats with disseminated tuberculous lesions. The main characteristics that differentiate isolates belonging to $M$. tuberculosis subsp. caprae from other members of the M. tuberculosis complex are a special combination of $p n c A$, $k a t G$ and gyrA gene polymorphisms that has not been found in other members of the complex, and specific 
fingerprinting patterns obtained from RFLP associated with IS6110, direct repeat and polymorphic GC-rich sequences, and direct variable repeat-spacer oligonucleotide typing (spoligotyping) that are very different to those obtained for other members.

Recently, Niemann et al. (2002) transferred M. tuberculosis subsp. caprae to the M. bovis group as Mycobacterium bovis subsp. caprae. The aim of this report is to present new genetic evidence for the taxon formerly named $M$. tuberculosis subsp. caprae, to refute this transfer. It should be noted that, although the name M. bovis subsp. caprae was validly published, according to Rule 27 (3) of the Bacteriological Code (Lapage et al., 1992), Mycobacterium bovis subsp. caprae (Aranaz et al. 1999) Niemann et al. 2002 is not valid because, at the time of publication, the type strain of the species was not deposited in two publicly accessible service collections in different countries (De Vos \& Trüper, 2000).

Phenotypic characteristics of the caprine mycobacterial isolates that differentiate them from $M$. bovis are a weak hydrolysis of Tween 80 after 10 days and sensitivity to pyrazinamide (PZA). Sensitivity to PZA has been used as a major criterion for distinguishing $M$. bovis from the other members of the M. tuberculosis complex. Growth of M. bovis strains is not inhibited by PZA, a key characteristic that was included by Karlson \& Lessel (1970) in the validation of the name and description of the species, while M. tuberculosis, M. africanum and M. microti are susceptible to this antimycobacterial drug. However, a small percentage of M. bovis strains sensitive to PZA was cited by Collins \& Yates (1981). Results of PZA susceptibility testing, depending on growth of the organisms when exposed to drugs, can be unreliable due to the technical difficulty in obtaining growth in the very acidic medium required for PZA activity (Davies et al., 2000; Hewlett et al., 1995). The test can vary depending on the method and media used, and can suffer from limited reproducibility and lack of reliability (Hannan et al., 2001).

The sequencing of the pyrazinamidase gene ( $p n c A)$ demonstrated a single $C \rightarrow G$ point mutation at nucleotide 169 that appears to be unique to M. bovis (Scorpio \& Zhang, 1996), while M. tuberculosis, M. africanum and M. microti harbour the functional wild-type $p n c A$ gene. The 'defective' $p n c A$ gene of $M$. bovis is a stable feature that has been used for the design of genetic tests for differentiation of M. bovis from M. tuberculosis (Scorpio et al., 1997; Espinosa de los Monteros et al., 1998). The sequence of the pncA gene of the type strain of M. tuberculosis subsp. caprae (CIP $105776^{\mathrm{T}}$ ) and seven other caprine mycobacterial isolates of four different spoligotyping profiles, and from different hosts, reveals that all isolates have the wild-type $p n c A$ gene. This result shows that the allele polymorphism $169 \mathrm{C}$ is a stable characteristic of the caprine isolates.

When the previously mentioned study of Collins \& Yates (1981) was carried out, DNA-based techniques were not as accessible as they are today for most average laboratories; thus, there are no data on the $p n c A$ and other gene sequences or on the spoligotyping profile of the strains used in their study, making it difficult to gather information on the real nature of the isolates examined by Collins \& Yates (1981).

Further differentiation amongst caprine mycobacterial isolates and M. bovis is drawn from the $g y r B$ gene sequence polymorphism analyses of several members of the $M$. tuberculosis complex from the studies of Kasai et al. (2000) and Niemann et al. (2000b). At nucleotide 1311, M. bovis and other members of the M. tuberculosis complex show a T, while caprine mycobacterial isolates show a $\mathrm{G}$; at position 1410, M. bovis has a characteristic $\mathrm{C} \rightarrow \mathrm{T}$ substitution, which was proposed by Kasai et al. (2000) for differentiation of the species, while caprine strains and the other members of the M. tuberculosis complex have a C (Niemann et al., 2000b). Sequencing of the gyrB gene from two Spanish caprine strains (the type strain of $M$. tuberculosis subsp. caprae plus an isolate from a domestic pig) and comparison to a sequence from the GenBank database (accession no. L27512; Kasai et al., 2000) demonstrated that these strains also had the specific 'caprine' polymorphism. These results increase the combination of gene polymorphisms specific for this unusual member of the M. tuberculosis complex.

Caprine mycobacterial isolates have special features by genetic fingerprinting. By direct variable repeat-spoligotyping, performed with first generation membranes following the method of Kamerbeek et al. (1997), they form a homogeneous cluster easily recognizable by the absence of spacers $1,3-16,30-33$ and 39-43. The lack of spacers 39-43 has also been described in M. bovis and M. microti. RFLP typing associated with IS6110, polymorphic GC-rich sequences and direct repeats also segregates caprine mycobacterial isolates from M. bovis isolates (Liébana et al., 1997; Aranaz et al., 1998).

Regarding its presence in other hosts, M. tuberculosis subsp. caprae was first isolated from goats, but it is not restricted to caprine herds. Predominant isolation of this pathogen from goats in our laboratory may reflect epidemiological facts (the main host species in Spain) and the high number of caprine herds we have studied. Until relatively recently, goat farming in Spain has remained as a traditional farming system with isolated populations, and herds have not been included in health schemes and eradication campaigns. We have also isolated caprine mycobacterial isolates from cattle, wild boar (Sus scrofa) and pigs under extensive management. As was commented on in the description of M. tuberculosis subsp. caprae (Aranaz et al., 1999), isolates that displayed the caprine spoligotyping pattern have also been found in human patients, and these clinical cases have been linked with goat farming (Gutiérrez et al., 1997).

Regarding the presence of $M$. tuberculosis subsp. caprae strains in other countries, the caprine spoligotype has also been identified in isolates from goats in France (Haddad et al., 2001) and similar spoligotypes have been reported in cattle and free-living red deer (Cervus elaphus) in Austria 
(Glawischnig et al., 2000). We suggest that the clinical $M$. bovis isolates from cattle and humans described by Niemann et al. (2000a, b) are likely to be caprine isolates, because they share features such as susceptibility to PZA (they had the wild-type $p n c A$ sequence) and the sequence of the gyrB gene, and the spoligotyping patterns were defined by the typical absence of spacers.

Further evidence for the independence of the caprine mycobacterial isolates from $M$. bovis is derived from two recent studies that have examined the evolution of the M. tuberculosis complex (Brosch et al., 2002; Mostowy et al., 2002). Both these studies tested two different and representative sets of isolates of the $M$. tuberculosis complex ( $n=100$ and $n=66$, respectively) from different hosts and countries. These isolates were tested for the presence/ absence of regions of difference (RD), expected to represent unidirectional genetic events, and single nucleotide polymorphism analysis of the $p n c A, g y r A$ and $k a t G$ genes was also done. Because the distribution of deletions suggests their order of occurrence during bacterial evolution, such studies propose an evolutionary scenario for the $M$. tuberculosis complex. In fact, the results of the $\mathrm{RD}$ analysis match those of the table of differential characteristics of the members of the complex (Table 1). Results show that ancestral $M$. tuberculosis strains and ' $M$. canettii' lack none of the studied deleted regions, and imply that the common ancestor for the M. tuberculosis complex would resemble $M$. tuberculosis or ' $M$. canettii'. Other classical members of the $M$. tuberculosis complex form a separate lineage. The first branch would be represented by M. africanum (lacks RD9), then M. microti/the seal bacillus (lack RD9, and also RD7, RD8 and RD10) and caprine isolates (lack RD9, RD7, RD8, RD10, and RD5, RD6, RD12, RD13 and N-RD25). Classical $M$. bovis shows the greatest number of $\mathrm{RD}$ deletions relative to other members of the M. tuberculosis complex (apart of the aforementioned regions, it also lacks RD4) and would be the last link of this lineage. This fact would mean that the group of caprine mycobacterial isolates (or its ancestor) is older than M. bovis (or its ancestor).

These findings reinforce the original suggestion that the caprine mycobacterial strains are a taxon of the M. tuberculosis complex, independent of M. bovis. From a strict taxonomic point of view, the caprine isolates should be considered as a subspecies of $M$. tuberculosis. We believe that the nomenclature ' $M$. tuberculosis' should prevail for the following reasons. First, there is compelling evidence

Table 1. Differential characteristics of the caprine mycobacterial isolates ( $M$. caprae) in comparison with other members of the M. tuberculosis complex

Species: 1, M. tuberculosis (classical); 2, M. tuberculosis (Asian); 3, M. africanum (type I); 4, M. africanum (type II); 5, M. microti; 6, M. caprae; 7, M. bovis; 8, M. bovis BCG. IS6110, IS1081 and MPB70 are present in all members listed (very occasionally, M. tuberculosis strains lack the former). Data were taken from references cited in the text. + , Positive test result; - , negative test result; $\mathrm{V}$, variable test result; $\mathrm{ND}$, no data available or insufficient information; S, sensitive; R, resistant.

\begin{tabular}{|c|c|c|c|c|c|c|c|c|}
\hline Characteristic & 1 & 2 & 3 & 4 & 5 & 6 & 7 & 8 \\
\hline Niacin accumulation & + & + & $\mathrm{V}$ & $\mathrm{V}$ & + & - & - & - \\
\hline $\mathrm{TCH}$ & $\mathrm{R}$ & s & s & s & s & $s \dagger$ & s & s \\
\hline Pyrazinamide & S & S & S & S & s & s & $\mathrm{R}$ & $\mathrm{R}$ \\
\hline pncA с 57 & CAC (His) & $\mathrm{CAC}$ & $\mathrm{CAC}$ & CAC & $\mathrm{CAC}$ & $\mathrm{CAC}$ & $\begin{array}{l}\text { GAC } \\
(\text { Asp) }\end{array}$ & GAC \\
\hline kat $G$ с 463 & $\begin{array}{c}\text { CGG (Arg) } \\
\text { CTG (Leu) }\end{array}$ & CGG/CTG & ND & ND & CTG & CTG & CTG & CTG \\
\hline$o x y R$ n 285 & G & G & G & G & G & A & A & $\mathrm{A}$ \\
\hline gyrA c 95 & $\begin{array}{l}\text { AGC (Ser) } \\
\text { ACC (Thr) }\end{array}$ & - & ACC & ACC & ACC & ACC & ACC & ACC \\
\hline
\end{tabular}

${ }^{*}$ Occasionally some isolates lack this element (Liébana et al., 1996).

$\dagger$ Resistant to 1 and $2 \mu \mathrm{g}$ 2-thiophenecarboxylic acid hydrazide (TCH) $\mathrm{ml}^{-1}$, but sensitive to 5 and $10 \mu \mathrm{g} \mathrm{TCH} \mathrm{ml}^{-1}$. 
that M. tuberculosis is the ancestor of the caprine mycobacterial taxon, or the member of the complex more related to it. Second, M. tuberculosis is the type species of the genus Mycobacterium and was the first member of the complex to be described. Third, M. tuberculosis is by far the most important pathogen of the complex in terms of the number of infected hosts and its public health implications. However, for an easy recognition of this unusual mycobacterial pathogen from goats by the health community, in terms of diagnosis and epidemiology, and within the current context of the existing nomenclature of the M. tuberculosis complex, we suggest the name Mycobacterium caprae for this organism.

Correct identification of mycobacteria is necessary for accurate diagnosis and appropriate treatment, and for epidemiological study in order to trace the index case or source of infection. Therefore, it is advisable that assignation to a 'species' or 'subspecies' should not be based exclusively on phenotypic characteristics when other tests can be performed with low cost and complexity. It is to be expected that additional techniques will become available in the future, and that they will help to achieve a more definitive classification of the these important pathogens. Such techniques should lead us further in the taxonomy of the M. tuberculosis complex.

\section{Description of Mycobacterium caprae comb. nov., sp. nov.}

Mycobacterium caprae (ca'p.rae. L. fem. gen. n. caprae referring to capra, the L. fem. n. for goat, the host animal from which the bacterium was first isolated).

The description is the same as that given for M. tuberculosis subsp. caprae (Aranaz et al., 1999), with the addition that isolates present a specific gyrB gene polymorphism: nucleotide 1311 is a $\mathrm{G}$ and nucleotide 1410 is a $\mathrm{C}$.

The type strain is $\mathrm{gM}-1^{\mathrm{T}}\left(=\mathrm{CIP} 105776^{\mathrm{T}}=\right.$ ATCC BAA$824^{\mathrm{T}}$ ), which has the characteristics described for the taxon.

\section{Acknowledgements}

A. Aranaz is the recipient of a fellowship from the Ramon y Cajal Programme (Spanish Ministry of Science and Technology/U.C.M.). This research has been partially funded by the Spanish Ministry of Agriculture, Fisheries and Food.

\section{References}

Aranaz, A., Liébana, E., Mateos, A., Domínguez, L. \& Cousins, D. (1998). Restriction fragment length polymorphism and spacer oligonucleotide typing: a comparative analysis of fingerprinting strategies for Mycobacterium bovis. Vet Microbiol 61, 311-324.

Aranaz, A., Liébana, E., Gómez-Mampaso, E. \& 8 other authors (1999). Mycobacterium tuberculosis subsp. caprae subsp. nov.: a taxonomic study of a new member of the Mycobacterium tuberculosis complex isolated from goats in Spain. Int J Syst Bacteriol 49, 1263-1273.
Brosch, R., Gordon, S. V., Marmiesse, M. \& 12 other authors (2002). A new evolutionary scenario for the Mycobacterium tuberculosis complex. Proc Natl Acad Sci U S A 99, 3684-3689.

Collins, C. H. \& Yates, M. D. (1981). A study of bovine strains of Mycobacterium tuberculosis isolated from humans in South-East England, 1977-1979. Tubercle 62, 113-116.

Cousins, D. V., Francis, B. R., Gow, B. L., Collins, D. M., McGlashan, C. H., Gregory, A. \& MacKenzie, R. M. (1990). Tuberculosis in captive seals: bacteriological studies on an isolate belonging to the Mycobacterium tuberculosis complex. Res Vet Sci 48, 196-200.

Cousins, D. V., Williams, S. N., Reuter, R., Forshaw, D., Chadwick, D., Coughran, D., Collins, P. \& Gales, N. (1993). Tuberculosis in wild seals and characterisation of the seal bacillus. Aust Vet J 70, 92-97.

Davies, A. P., Billington, O. J., McHugh, T. D., Mitchison, D. A. \& Gillespie, S. H. (2000). Comparison of phenotypic and genotypic method for pyrazinamide susceptibility testing with Mycobacterium tuberculosis. J Clin Microbiol 38, 3686-3688.

De Vos, P. \& Trüper, H. G. (2000). Judicial Commission of the International Committee on Systematic Bacteriology. IXth International (IUMS) Congress of Bacteriology and Applied Microbiology. Minutes of the meetings, 14, 15 and 18 August 1999, Sydney, Australia. Int J Syst Evol Microbiol 50, 2239-2244.

Espinosa de los Monteros, L. E., Galán, J. C., Gutiérrez, M. \& 8 other authors (1998). Allele-specific PCR method based on pncA and $\operatorname{oxyR}$ sequences for distinguishing Mycobacterium bovis from Mycobacterium tuberculosis: intraspecific $M$. bovis pncA sequence polymorphism. J Clin Microbiol 36, 239-242.

Glawischnig, W., Prodinger, W. M. \& Schöpf, K. (2000). Bovine tuberculosis in free-living Austrian red deer (Cervus elaphus): epidemiological investigation using DNA-fingerprinting. In Abstracts of the 4th Meeting of the European Wildlife Disease Association, Zaragoza (Spain), 20-23 September, 2000. Zaragoza: European Wildlife Disease Association.

Gutiérrez, M., Samper, S., Jiménez, M. S., van Embden, J. D. A., Marin, J. F. \& Martín, C. (1997). Identification by spoligotyping of a caprine genotype in Mycobacterium bovis strains causing human tuberculosis. J Clin Microbiol 35, 3328-3330.

Haddad, N., Ostyn, A., Karoui, C., Masselot, M., Thorel, M. F., Hughes, S. L., Inwald, J., Hewinson, R. G. \& Durand, B. (2001). Spoligotype diversity of Mycobacterium bovis strains isolated in France from 1979 to 2000. J Clin Microbiol 39, 3623-3632.

Hannan, M. M., Desmond, E. P., Morlock, G. P., Mazurek, G. H. \& Crawford, J. T. (2001). Pyrazinamide-monoresistant Mycobacterium tuberculosis in the United States. J Clin Microbiol 39, 647-650.

Hewlett, D., Jr, Horn, D. L. \& Alfalla, C. (1995). Drug-resistant tuberculosis: inconsistent results of pyrazinamide susceptibility testing. JAMA 273, 916-917.

Kamerbeek, J., Schouls, L., Kolk, A. \& 8 other authors (1997). Simultaneous detection and strain differentiation of Mycobacterium tuberculosis for diagnosis and epidemiology. J Clin Microbiol 35, 907-914.

Karlson, A. G. \& Lessel, E. F. (1970). Mycobacterium bovis nom. nov. Int J Syst Bacteriol 20, 273-282.

Kasai, H., Ezaki, T. \& Harayama, S. (2000). Differentiation of phylogenetically related slowly growing mycobacteria by their gyrB sequences. J Clin Microbiol 38, 301-308.

Lapage, S. P., Sneath, P. H. A., Lessel, E. F., Skerman, V. B. D., Seeliger, H. P. R. \& Clark, W. A. (editors) (1992). International Code of Nomenclature of Bacteria (1990 Revision). Bacteriological Code. Washington, DC: American Society for Microbiology. 
Liébana, E., Aranaz, A., Francis, B. \& Cousins, D. (1996). Assessment of genetic markers for species differentiation within the Mycobacterium tuberculosis complex. J Clin Microbiol 34, 933-938.

Liébana, E., Aranaz, A., Domínguez, L., Mateos, A., GonzalezLlamazares, O., Rodríguez-Ferri, E. F., Domíngo, M., Vidal, D. \& Cousins, D. (1997). The insertion element IS6110 is a useful tool for DNA fingerprinting of Mycobacterium bovis isolates from cattle and goats in Spain. Vet Microbiol 54, 223-233.

Mostowy, S., Cousins, D., Brinkman, J., Aranaz, A. \& Behr, M. (2002). Genomic deletions suggest a phylogeny for the Mycobacterium tuberculosis complex. J Infect Dis 186, 74-80.

Niemann, S., Richter, E. \& Rüsch-Gerdes, S. (2000a). Differentiation among members of the Mycobacterium tuberculosis complex by molecular and biochemical features: evidence for two pyrazinamidesusceptible subtypes of M. bovis. J Clin Microbiol 38, 152-157.

Niemann, S., Harmsen, D., Rüsch-Gerdes, S. \& Richter, E. (2000b). Differentiation of clinical Mycobacterium tuberculosis complex isolates by $\operatorname{gyr} B$ DNA sequence polymorphism analysis. J Clin Microbiol 38, 3231-3234.

Niemann, S., Richter, E. \& Rüsch-Gerdes, S. (2002). Biochemical and genetic evidence for the transfer of Mycobacterium tuberculosis subsp. caprae Aranaz et al. 1999 to the species Mycobacterium bovis
Karlson and Lessel 1970 (Approved Lists 1980) as Mycobacterium bovis subsp. caprae comb. nov. Int J Syst Evol Microbiol 52, 433-436.

Scorpio, A. \& Zhang, Y. (1996). Mutations in $p n c A$, a gene encoding pyrazinamidase/nicotinamidase, cause resistance to antituberculous drug pyrazinamide in tubercle bacillus. Nat Med 2, 662-667.

Scorpio, A., Collins, D., Whipple, D., Cave, D., Bates, J. \& Zhang, Y. (1997). Rapid differentiation of bovine and human tubercle bacilli based on a characteristic mutation in the bovine pyrazinamidase gene. J Clin Microbiol 35, 106-110.

van Soolingen, D., Hoogenboezem, T., de Haas, P. E. W. \& 9 other authors (1997). A novel pathogenic taxon of the Mycobacterium tuberculosis complex, Canetti: characterization of an exceptional isolate from Africa. Int J Syst Bacteriol 47, 1236-1245.

Wayne, L. G. (1984). Mycobacterial speciation. In The Mycobacteria: a Sourcebook, pp. 25-65. Edited by G. P. Kubica \& L. G. Wayne. New York: Marcel Dekker.

Wayne, L. G. \& Kubica, G. P. (1986). Family Mycobacteriaceae Chester 1897, 63 ${ }^{\mathrm{AL}}$. In Bergey's Manual of Systematic Bacteriology, vol. 2, pp. 1436-1457. Edited by P. H. A. Sneath, N. S. Mair, M. E. Sharpe \& J. G. Holt. Baltimore: Williams \& Williams. 